\title{
Irrationality measures for some automatic real numbers
}

\author{
Boris Adamczewski and Tanguy Rivoal
}

\begin{abstract}
This paper is devoted to the rational approximation of automatic real numbers, that is, real numbers whose expansion in an integer base can be generated by a finite automaton. We derive upper bounds for the irrationality exponent of famous automatic real numbers associated with the Thue-Morse, Rudin-Shapiro, paperfolding and Baum-Sweet sequences. These upper bounds arise from the construction of some explicit Padé or Padé type approximants for the generating functions of these sequences. In particular, we prove that the Thue-Morse-Mahler numbers have an irrationality exponent at most equal to 4 . We also obtain an explicit description of infinitely many convergents to these numbers.
\end{abstract}

\section{Introduction}

A real number is said to be generated by a finite automaton, or simply automatic, if for some integer $b \geqslant 2$ its $b$-ary expansion can be produced by a finite automaton. Automatic real numbers form a distinguished class among computable numbers lying at the lowest level of the hierarchy arising from Turing machines. We refer the reader to the monograph [5] for a formal definition and a more complete introduction to finite automata and automatic numbers (in particular, a whole chapter is devoted to these numbers).

While emblematic examples of automatic numbers, such as

$$
\xi_{d}=\sum_{n \geqslant 1} \frac{1}{10^{d^{n}}},
$$

have been known to be transcendental for a long time (see for instance [11, 14]), the algebraic nature of all automatic number was established only recently in [1]: irrational automatic real numbers are all transcendental. We recall that rational numbers are automatic. Once irrationality or transcendence of a number is proved, it is natural to continue investigating its diophantine properties. For example, it was conjectured that automatic irrational numbers are all $S$-numbers in Mahler's classification. The approach of [1] was then further developped in [2] to provide, amongst other things, a first step towards the solution of this problem.

In this paper, we are interested in rational approximations to automatic real numbers. We recall that the irrationality exponent $\mu(\xi)$ (also sometimes called the irrationality measure) of a real irrational number $\xi$ is the infinum of the real numbers $\mu$ such that the inequality

$$
\left|\xi-\frac{p}{q}\right|>\frac{1}{q^{\mu}}
$$

admits finitely many solutions $(p, q) \in \mathbb{Z} \times \mathbb{N}^{\times}$. If no such $\mu$ exists, we say that $\mu(\xi)=+\infty$, which defines the class of Liouville numbers. Eq. (1) provides an irrationality measure for $\xi$. The convergents of the continued fraction of $\xi$ imply that $\mu(\xi) \geqslant 2$ for every irrational number $\xi$. The

2000 Mathematics Subject Classification 11J82 (primary), 11B85 (secondary).

Keywords: Automatic sequences, irrationality measures, Padé approximants

B. A. is supported by the ANR through the project "DyCoNum"-JCJC06_134288. 


\section{Boris AdAmczewsiki AND TANGuy Rivoal}

irrationality exponent of almost all real numbers $\left({ }^{1}\right)$ is exactly equal to 2 . A systematic study of rational approximations to automatic irrational numbers was started in [4]. These authors made the approach of $[1,3]$ quantitative in order to prove that automatic numbers all have a finite irrationality exponent, thereby confirming a conjecture of Shallit [16]. In other words, such numbers are never Liouville numbers.

All the above mentioned results or conjectures show that automatic numbers share (or are expected to share) some properties satisfied by almost all real numbers, such as being transcendental, having a finite irrationality exponent, or being an $S$-number. In contrast, it is known that some automatic real numbers are very special with respect to rational approximation. As an illustration, we recall that $\mu\left(\xi_{d}\right)=d$ for every integer $d \geqslant 2$. Very recently, Bugeaud [6] tackled this question and proved that for every rational number $\omega$ greater than or equal to 2 there are infinitely many automatic real numbers with an irrationality exponent equal to $\omega\left({ }^{2}\right.$.). Actually, Bugeaud exhibits examples of suitable lacunary series for which he can compute exactly the irrationality exponent. However, these examples are somewhat ad hoc and are not chosen amongst the most famous automatic numbers, such as those constructed with Thue-Morse, Rudin-Shapiro, paperfolding or Baum-Sweet sequences. It is in fact unclear how such particular automatic numbers can be approximated by rationals, and, most of the time, no good upper bounds for their irrationality exponent are known.

In this paper, we study this question and derive upper bounds for the irrationality exponent of all the automatic real numbers mentioned just above (see Theorem 4.2 in Section 4). These bounds are deduced from the explicit construction of Padé and Padé type approximants for the generating functions of these automatic sequences. These functional approximations are obtained in two different ways. The first approach is based on functional equations satisfied by the generating functions and falls within the range of Mahler's transcendence method (see [14]). It provides Padé approixmants (see Section 2) which are good enough to provide irrationality measures. The second approach is the one started in [4] and relies on a classical theorem of Cobham. Most of the time, it only provides Padé type approximants, but still good enough to get irrationality measures (see Section 3). It also sometimes leads to the construction of "real" Padé approximants (see the discussion in 3.3). Our irrationality measures are deduced in Subsections 4.2 and 4.3. In Subsection 4.4, our Theorem 4.2 is the result of the comparison of the measures produced by both methods. To give an idea of these measures, let us mention the case of the emblematic Thue-Morse-Mahler numbers $\mathbf{T}_{2}(1 / b):=\sum_{n=1}^{\infty} t_{n} b^{n-1}$ generated by the Thue-Morse sequence $\mathbf{t}=\left(t_{n}\right)_{n \geqslant 0}$, that is, $t_{n}=1$ if the sum of the binary digits of $n$ is odd and $t_{n}=0$ otherwise. The transcendence of these numbers was first proved by Mahler in 1929 [14]. We prove in this paper that

$$
\mu\left(\mathbf{T}_{2}(1 / b)\right) \leqslant 4
$$

for any integer $b \geqslant 2$, which improves on the previously known bound 5 obtained in [4]. We believe that our method might lead to the bound 3 for these numbers: our reasons for this are given in Section 5. Our method also enables us to produce an explicit subsequence of the convergents of $\mathbf{T}_{2}(1 / b)$ for any $b \geqslant 2$; this is Theorem 5.1 in Section 5 .

\footnotetext{
${ }^{1}$ Here and troughout the rest of this paper, "almost all" refers to Lebesgue measure.

${ }^{2}$ In view of this result, it would be interesting to know wether or not the irrationality exponent of an automatic real number is always a rational number. Note that automatic real numbers form a countable set and the values of their irrationality exponent can thus not reach all real numbers greater than or equal to 2
} 


\section{IRRATIONALITY MEASURES FOR SOME AUTOMATIC REAL NUMBERS}

\section{Padé approximants of generating functions of automatic sequences}

In this section, we provide a number of examples of automatic sequences $\left(a_{n}\right)_{n \geqslant 0}$ such that we can compute explicitely certain Padé approximants of their generating function $\sum_{n=0}^{\infty} a_{n} z^{n}$.

We briefly recall that given a field $\mathbb{K}$, a formal power series $F(x) \in \mathbb{K}[[z]]$ and two integers $p, q \geqslant 0$, the Padé approximant $[p / q]_{F}(z)$ is any rational fraction $A(z) / B(z) \in \mathbb{K}(z)$ such that $\operatorname{deg}(A) \leqslant p, \operatorname{deg}(B) \leqslant q$ and $\operatorname{ord}_{z=0}(B(z) F(z)-A(z)) \geqslant p+q+1$. It turns out that while the pair $(A, B)$ has no reason to be unique (not even to a multiplicative constant), the fraction $A / B$ is unique. See [9] for details.

It is well-known that when $\mathbb{K}=\mathbb{Q}$ or a number field, such approximations are useful for obtaining irrationality or transcendental results for the values of $F(x)$ at certain rational numbers $z$. Accordingly, we will use in Subsection 4.2 the contructions of this section to derive irrationality measures for the corresponding automatic numbers.

\subsection{Thue-Morse like sequences}

The Thue-Morse sequence $\mathbf{t}=\left(t_{n}\right)_{n \geqslant 0}$ is defined by $t_{0}=0, t_{2 n}=t_{n}$ et $t_{2 n+1}=1-t_{n}$. We can generalise it as follows: for any integer $k \geqslant 2$, we define the sequence $\mathbf{t}_{k}=\left(t_{n}^{(k)}\right)_{n \geqslant 0}$ by $t_{0}^{(k)}=0$, $t_{k n+1}^{(k)}=1-t_{n}^{(k)}$ and $t_{k n+j}^{(k)}=t_{n}^{(k)}$ for $j=2, \ldots, k-1$. We define the generating function of $\mathbf{t}_{k}$ by

$$
\mathbf{T}_{k}(z)=\sum_{n=0}^{\infty} t_{n}^{(k)} z^{n-1} \in \mathbb{Z}[[z]] .
$$

The case $k=3$ is special because in this case $t_{n}^{(k)}=\left(1+(-1)^{n+1}\right) / 2$ for every $n \geqslant 0$ and $\mathbf{T}_{3}(z)=$ $1 /\left(1-z^{2}\right)$. We will show below that this is the only case where $\mathbf{T}_{k}(z) \in \mathbb{Q}(z)$.

From the definition of $\mathbf{t}_{k}$, it is easy to prove that $\mathbf{T}_{k}$ satisfies the functional equation:

$$
z^{k-1} \mathbf{T}_{k}\left(z^{k}\right)=\frac{z-1}{\sigma_{k}(z)} \mathbf{T}_{k}(z)-\frac{z-1}{\left(1-z^{k}\right) \sigma_{k}(z)},
$$

where $\sigma_{k}(z):=z^{k}-1+2 z-2 z^{2}$. This equation will be used in the proof of the following proposition, where we sometimes omit the parameter $k$ for readability's sake.

Proposition 2.1. For any integer $k \geqslant 2$, set $\widehat{k}=3$ if $k=2, \widehat{k}=1$ if $k=3$ and $\widehat{k}=k$ otherwise. For any integer $n \geqslant 0$, the Padé approximant $\left[\widehat{k} \cdot k^{n}-1 / 3 \cdot k^{n}-1\right]_{\mathbf{T}_{k}}$ of $\mathbf{T}_{k}$, denoted by $P_{n} / Q_{n}$, can be computed recursively as follows:

$$
\left\{\begin{array}{l}
Q_{n}(z)=(1-z)\left(1+z^{k^{n}}\right) \in \mathbb{Z}[z] \\
P_{0}(z)=1-z^{2}+z^{k-1}, \\
P_{n+1}(z)=\frac{1}{1-z^{k}} Q_{n+1}(z)-\frac{z^{k-1} \sigma_{k}(z)}{1-z^{k}} P_{n}\left(z^{k}\right), \\
R_{n}(z):=Q_{n}(z) \mathbf{T}_{k}(z)-P_{n}(z)=R_{0}\left(z^{k^{n}}\right) \prod_{j=1}^{n}\left(\frac{z^{(k-1) k^{j-1}} \sigma_{k}\left(z^{k^{j-1}}\right)}{z^{k^{j-1}}-1}\right) .
\end{array}\right.
$$

If $k=2, \operatorname{deg}\left(P_{n}\right)=3 \cdot 2^{n}-1, R_{0}(z)=-z^{5}+\mathcal{O}\left(z^{6}\right)$ and $\operatorname{ord}_{z=0}\left(R_{n}\right)=6 \cdot 2^{n}-1$.

If $k=3, P_{n}(z)=Q_{n}(z) /\left(1-z^{2}\right), \operatorname{deg}\left(P_{n}\right)=3^{n}-1$ and $R_{n}(z) \equiv 0$.

If $k \geqslant 4, \operatorname{deg}\left(P_{n}\right)=k^{n+1}-1, R_{0}(z)=z^{k+2}+\mathcal{O}\left(z^{k+3}\right)$ and $\operatorname{ord}_{z=0}\left(R_{n}\right)=(k+3) k^{n}-1$.

In all cases, $P_{n}(z) \in \mathbb{Z}[z]$.

Remarks 1. (a) It is not obvious from the above recursion that $P_{n}$ is a polynomial.

(b) The degree of $Q_{n}$ is $k^{n}+1$, which is much smaller than the maximum allowed in the 


\section{Boris AdAmczewsiki and TANguy Rivoal}

definition of $\left[\widehat{k} \cdot k^{n}-1 / 3 \cdot k^{n}-1\right]_{\mathbf{T}_{k}}$. In other words, we have also computed the Padé approximants $\left[\widehat{k} \cdot k^{n}-1 / k^{n}+1+\ell\right]_{\mathbf{T}_{k}}$ for every $\ell \in\left\{0,1, \ldots, 2 \cdot k^{n}-1\right\}$.

(c) Assume that $k \neq 3$. The expression of $R_{n}$ as a product shows that it cannot be 0 because $R_{0}$ is not identically 0 . It follows that $\mathbf{T}_{k}$ is not a rational fraction when $k \neq 3$. If it were, $\mathbf{T}_{k}=A / B$ say, a comparison of the degree and order at zero of the polynomial $B R_{n}=Q_{n} A-B P_{n}$ would give us that $R_{n}=0$ identically for $n \gg 1$, contradicting the above remark.

(d) The proof is in fact a check. Indeed, the results were guessed after some numerical computations for small values of $n$ and $k$. This is also the case of the other results stated and proved in this section.

Proof. First step. We first prove that the value $P_{0}(z)=1-z^{2}+z^{k-1}$ and the equation

$$
P_{n+1}(z)=\frac{1}{1-z^{k}} Q_{n+1}(z)-\frac{z^{k-1} \sigma_{k}(z)}{1-z^{k}} P_{n}\left(z^{k}\right)
$$

define a sequence of polynomials $P_{n}(z) \in \mathbb{Z}[[z]]$ of degree $\widehat{k} \cdot k^{n}-1$ such that $P_{n}(1)=1$.

We proceed by induction on $n \geqslant 0$. The hypothesis is trus for $n=0$ and we assume that it is true for some given $n$. We can therefore write $P_{n}(z)=1+(1-z) \widehat{P}_{n}(z)$ for a certain polynomial $\widehat{P}_{n}(z) \in \mathbb{Z}[[z]]$. Then

$$
\begin{aligned}
& \frac{1}{1-z^{k}} Q_{n+1}(z)-\frac{z^{k-1} \sigma_{k}(z)}{1-z^{k}} P_{n}\left(z^{k}\right) \\
& \quad=\frac{(1-z)\left(1+z^{k^{n+1}}\right)-z^{k-1} \sigma_{k}(z)}{1-z^{k}}-\frac{\sigma_{k}(z) z^{k-1}\left(1-z^{k}\right) \widehat{P}_{n}\left(z^{k}\right)}{1-z^{k}} .
\end{aligned}
$$

The right-most fraction obviously simplifies and gives a polynomial in $z$ with integer coefficients. Furthermore, after simplification (recall that $\sigma_{k}(z)=z^{k}-1+2 z-2 z^{2}$ ), we have that

$$
\frac{(1-z)\left(1+z^{k^{n+1}}\right)-z^{k-1} \sigma_{k}(z)}{1-z^{k}}=\frac{(1-z)\left(1+z^{k^{n+1}}-2 z^{k}\right)}{1-z^{k}}+z^{k-1} .
$$

Obviously, any $k$-th root of unity is also a root of $1+z^{k^{n+1}}-2 z^{k}$, which implies that the fraction on the right hand side of (4) is also a polynomial in $z$ with integer coefficients.

Therefore, the expression $P_{n+1}(z)$ defined by (3) is a polynomial in $z$ with integer coefficients. This equation also immediately yields that $P_{n+1}(1)=1$ under the inductive assumption that $P_{n}(1)=1$ because $Q_{n+1}(z)$ and $\sigma_{k}(z)$ both vanish at $z=1$, making simple to compute the value of the right hand side of (3) at $z=1$. The assertion on the degree is also easy to prove from (3): indeed,

$$
\begin{aligned}
\operatorname{deg}\left(\frac{1}{1-z^{k}} Q_{n+1}(z)\right) & =k^{n+1}-k+1 \\
\operatorname{deg}\left(\frac{(1-z)\left(1+z^{k^{n+1}}\right)-z^{k-1} \sigma_{k}(z)}{z^{k}-1}\right) & =k^{n+1}-k+1, \\
\operatorname{deg}\left(\sigma_{k}(z) z^{k-1} \widehat{P}_{n}\left(z^{k}\right)\right) & =\widehat{k} \cdot k^{n+1}-1,
\end{aligned}
$$

where the last equality holds because, by the inductive hypothesis, we have $\operatorname{deg}\left(\widehat{P}_{n}\right)=\widehat{k} \cdot k^{n}-2$. Thus $\operatorname{deg}\left(P_{n+1}\right)=\widehat{k} \cdot k^{n+1}-1$.

Second step. For $k=2, \mathbf{T}_{2}(z)=1+z+z^{3}+\mathcal{O}\left(z^{6}\right)$. For $k=3, \mathbf{T}_{3}(z)=1 /\left(1-z^{2}\right)$. For $k \geqslant 4$, $\mathbf{T}_{k}(z)=1+z^{k-1}+z^{k+1}+z^{k+2}+\mathcal{O}\left(z^{k+3}\right)$. By a straightforward computation, it then follows that the Padé approximant $[\widehat{k}-1 / 2]_{\mathbf{T}_{k}}$ is equal to $P_{0}(z) / Q_{0}(z)$ with $P_{0}(z)$ and $Q_{0}(z)$ as given by the theorem. 
In particular, $R_{0}(z)=-z^{5}+\mathcal{O}\left(z^{6}\right)(k=2), R_{0}(z)=0(k=3)$ and $R_{0}(z)=z^{k+2}+\mathcal{O}\left(z^{k+3}\right)$ $(k \geqslant 4)$.

We now prove by induction on $n \geqslant 0$ that $R_{n}(z):=Q_{n}(z) \mathbf{T}_{k}(z)-P_{n}(z)$ is equal to

$$
R_{0}\left(z^{k^{n}}\right) \prod_{j=1}^{n}\left(\frac{z^{(k-1) k^{j-1}} \sigma_{k}\left(z^{k^{j-1}}\right)}{z^{k^{j-1}}-1}\right)
$$

This will imply that $\operatorname{ord}_{z=0}\left(R_{n}\right)=(\widehat{k}+3) \cdot k^{n}-1$ and therefore that $P_{n} / Q_{n}$ is the Padé approximant $\left[\widehat{k} \cdot k^{n}-1 / 3 \cdot k^{n}-1\right]_{\mathbf{T}_{k}}$ of $\mathbf{T}_{k}$.

For $n=0$, there is nothing to prove. Let us assume that the hypothesis holds for some $n$. Using the trivial relation $Q_{n+1}(z)=\frac{1-z}{1-z^{k}} Q_{n}\left(z^{k}\right)$, Eq. (3) and the functional equation (2), we see that

$$
\begin{aligned}
& Q_{n+1}(z) \mathbf{T}_{k}(z)-P_{n+1}(z) \\
& =\frac{1-z}{1-z^{k}} Q_{n}\left(z^{k}\right)\left(\frac{z^{k-1} \sigma_{k}(z)}{z-1} \mathbf{T}_{k}\left(z^{k}\right)+\frac{1}{1-z^{k}}\right)-\frac{1-z}{\left(1-z^{k}\right)^{2}} Q_{n}\left(z^{k}\right)+\frac{z^{k-1} \sigma_{k}(z)}{1-z^{k}} P_{n}\left(z^{k}\right) \\
& =\frac{z^{k-1} \sigma_{k}(z)}{z^{k}-1}\left(Q_{n}\left(z^{k}\right) \mathbf{T}_{k}\left(z^{k}\right)-P_{n}\left(z^{k}\right)\right) \\
& =\frac{z^{k-1} \sigma_{k}(z)}{z^{k}-1} R_{0}\left(z^{k^{n+1}}\right) \prod_{j=1}^{n}\left(\frac{z^{(k-1) k^{j}} \sigma_{k}\left(z^{k^{j}}\right)}{z^{k^{j}}-1}\right) \\
& =R_{0}\left(z^{k^{n+1}}\right) \prod_{j=1}^{n+1}\left(\frac{z^{(k-1) k^{j-1}} \sigma_{k}\left(z^{k^{j-1}}\right)}{z^{k^{j-1}}-1}\right),
\end{aligned}
$$

where we have used the inductive assumption in the penultimate equality.

It is now easy to compute the order at zero of $R_{n}(z)$ because we can rewrite the product as

$$
R_{n}(z)=R_{0}\left(z^{k^{n}}\right) z^{k^{n}-1} \prod_{j=1}^{n+1} \frac{\sigma_{k}\left(z^{k^{j-1}}\right)}{z^{k^{j-1}}-1},
$$

where $\frac{\sigma_{k}\left(z^{k^{j-1}}\right)}{z^{k^{j-1}}-1}$ does not vanish at $z=0$. When $k=3$, we have $R_{n}(z)=0$ while if $k \neq 3$ we deduce that $\operatorname{ord}_{z=0}\left(R_{n}\right)=(\widehat{k}+3) \cdot k^{n}-1$ since ord $\operatorname{or}_{z=0}\left(R_{0}\right)=\widehat{k}+2$.

\subsection{The Rudin-Shapiro sequence}

The Rudin-Shapiro sequence $\mathbf{r}=\left(r_{n}\right)_{n \geqslant 0}$ is defined by $r_{0}=1, r_{2 n}=r_{n}$ and $r_{2 n+1}=(-1)^{n} r_{n}$. Its generating function

$$
\mathbf{R}(z)=\sum_{n=0}^{\infty} r_{n} z^{n}
$$

satisfies the functional equation $\mathscr{R}(z)=\mathscr{R}\left(z^{2}\right)+z \mathscr{R}\left(-z^{2}\right)$.

Proposition 2.2. For any integer $n \geqslant 1$, the Padé approximant $\left[\frac{5}{2} \cdot 2^{n}-1 / 2^{n}\right]_{\mathbf{R}}$ of $\mathbf{R}$, denoted by $P_{n} / Q_{n}$ can be computed recursively as follows:

$$
\left\{\begin{array}{l}
Q_{n}(z)=1+z^{2^{n}} \in \mathbb{Z}[z], \\
P_{n+1}(z)=P_{n}\left(z^{2}\right)+z P_{n}\left(-z^{2}\right), \\
R_{n+1}(z)=R_{n}\left(z^{2}\right)+z R_{n}\left(-z^{2}\right),
\end{array}\right.
$$

where $R_{n}(z):=Q_{n}(z) \mathbf{R}(z)-P_{n}(z), P_{1}(z)=1+z+2 z^{2}+2 z^{4}, R_{1}(z)=\left(1+z^{2}\right) \mathbf{R}(z)-(1+z+$ $\left.2 z^{2}+2 z^{4}\right)=2 z^{7}+\mathcal{O}\left(z^{8}\right)$. The polynomial $P_{n}(z) \in \mathbb{Z}[z]$ is of degree $\frac{5}{2} \cdot 2^{n}-1$. The order at zero of $R_{n}$ is $\frac{7}{2} \cdot 2^{n}$. 


\section{Boris AdAmczewsiki AND TANGuY Rivoal}

Remarks 2. (a) Contrary to the situation of Theorem 2.1, the polynomials $P_{n}$ and $Q_{n}$ in Theorem 2.2 have the maximal degree allowed by the definition of $\left[\frac{5}{2} \cdot 2^{n}-1 / 2^{n}\right]_{\mathbf{R}}$.

(b) It is also possible to describe explicitly the Padé approximant $\left[\frac{9}{2} \cdot 2^{n}-1 / 2^{n}\right]_{\mathbf{R}}$, whose denominator is $1-z^{2^{n}}$. But it seems less interesting for diophantine applications (see below).

Proof. The case $n=1$ can be checked at hand.

We note that $Q_{n}(z)=Q_{n}(-z)$ and $Q_{n+1}(z)=Q_{n}\left(z^{2}\right)$, which will be used below. By induction on $n \geqslant 1$, one checks (a) that the polynomial $P_{n}$ is of degree $\frac{5}{2} \cdot 2^{n}-1$ with integer coefficients, and (b) that the function $\widehat{R}_{n}$ defined by the recursion $\widehat{R}_{n+1}(z)=\widehat{R}_{n}\left(z^{2}\right)+z \widehat{R}_{n}\left(-z^{2}\right), \widehat{R}_{1}=R_{1}$, has order $\frac{7}{2} \cdot 2^{n}$ at zero.

We now proceed to prove by induction on $n \geqslant 1$ that $\widehat{R}_{n}=R_{n}$, where $R_{n}:=Q_{n} \mathbf{R}-P_{n}$. This will show that $P_{n} / Q_{n}$ is the Padé approximant we are looking for. This is true for $n=1$ by definition. Let us assume that it is true for a given $n$. Then, we have the chain of equalities

$$
\begin{aligned}
\widehat{R}_{n+1}(z) & =\widehat{R}_{n}\left(z^{2}\right)+z \widehat{R}_{n}\left(-z^{2}\right)=R_{n}\left(z^{2}\right)+z R_{n}\left(-z^{2}\right) \\
& =Q_{n}\left(z^{2}\right) \mathbf{R}\left(z^{2}\right)-P_{n}\left(z^{2}\right)+z Q_{n}\left(-z^{2}\right) \mathbf{R}\left(-z^{2}\right)-P_{n}\left(-z^{2}\right) \\
& =Q_{n+1}(z)\left(\mathbf{R}\left(z^{2}\right)+z \mathbf{R}\left(-z^{2}\right)\right)-\left(P_{n}\left(z^{2}\right)+z P_{n}\left(-z^{2}\right)\right) \\
& =Q_{n+1}(z) \mathbf{R}(z)-P_{n+1}(z)=R_{n+1}(z),
\end{aligned}
$$

where we have used the functional equation satisfied by $\mathbf{R}$.

\subsection{The paperfolding sequence}

The paperfolding sequence $\mathbf{f}=\left(f_{n}\right)_{n \geqslant 0}$ is defined by $f_{4 n}=1, f_{4 n+2}=-1, f_{4 n+1}=f_{2 n}$ and $f_{4 n+3}=f_{2 n+1}$. Its generating series

$$
\mathbf{F}(z)=\sum_{n=0}^{\infty} f_{n} z^{n}
$$

satisfies the functional equation $z \mathscr{F}\left(z^{2}\right)=\mathscr{F}(z)-\frac{1}{1+z^{2}}$.

Proposition 2.3. For any integer $n \geqslant 1$, the Padé approximant $\left[3\left(2^{n}-1\right) / 2\left(2^{n}-1\right)\right]_{\mathbf{F}}$ of $\mathbf{F}$, denoted by $P_{n} / Q_{n}$ can be computed recursively as follows

$$
\left\{\begin{array}{l}
Q_{n}(z)=\prod_{j=1}^{n}\left(1+z^{2^{j}}\right) \in \mathbb{Z}[z], \\
P_{n+1}(z)=z\left(1+z^{2}\right) P_{n}\left(z^{2}\right)+Q_{n}\left(z^{2}\right), \\
R_{n+1}(z)=z\left(1+z^{2}\right) R_{n}\left(z^{2}\right)=z^{2^{n-1}-1} R_{1}\left(z^{2^{n-1}}\right) \prod_{j=1}^{n-1}\left(1+z^{2^{j}}\right),
\end{array}\right.
$$

where $R_{n}(z):=Q_{n}(z) \mathscr{F}(z)-P_{n}(z), P_{1}(z)=1+z+2 z^{3}, R_{1}(z)=\left(1+z^{2}\right) \mathscr{F}(z)-\left(1+z+2 z^{3}\right)=$ $2 z^{9}+\mathcal{O}\left(z^{10}\right)$. The polynomial $P_{n}$ has integer coefficients and has degree $3\left(2^{n}-1\right)$. The order at zero of $R_{n}$ is $5\left(2^{n}-1\right)+4$.

Proof. The case $n=1$ can be checked by hand.

For future use, note that $Q_{n+1}(z)=\left(1+z^{2}\right) Q_{n}\left(z^{2}\right)$. One checks by induction on $n \geqslant 1$ that (a) the polynomial $P_{n}$ has degree $3 \cdot 2^{n}-3$ and integer coefficients, and (b) the function $\widehat{R}_{n}$ defined by the recursion $\widehat{R}_{n+1}(z)=z\left(1+z^{2}\right) \widehat{R}_{n}\left(z^{2}\right), \widehat{R}_{1}=R_{1}$, has order at zero equal to $5 \cdot 2^{n}-1$.

We now show by induction on $n \geqslant 1$ that $\widehat{R}_{n}=R_{n}$, where $R_{n}:=Q_{n} \mathscr{F}-P_{n}$. This will prove that $P_{n} / Q_{n}$ is the Padé approximant we are looking for. This is true for $n=1$ by definition. Let us 


\section{IRRATIONALITY MEASURES FOR SOME AUTOMATIC REAL NUMBERS}

assume that this is true for a given $n$. Then we have the following chain of equalities

$$
\begin{aligned}
\widehat{R}_{n+1}(z) & =z\left(1+z^{2}\right) \widehat{R}_{n}\left(z^{2}\right)=z\left(1+z^{2}\right) R_{n}\left(z^{2}\right) \\
& =z\left(1+z^{2}\right)\left(Q_{n}\left(z^{2}\right) \mathbf{F}\left(z^{2}\right)-P_{n}\left(z^{2}\right)\right) \\
& =Q_{n+1}(z) \mathbf{F}(z)-Q_{n}\left(z^{2}\right)-z\left(1+z^{2}\right) P_{n}\left(z^{2}\right) \\
& =Q_{n+1}(z) \mathbf{F}(z)-P_{n+1}(z)=R_{n+1}(z),
\end{aligned}
$$

where we have used the functional equation satisfied by $\mathbf{F}$.

The expression of $R_{n}(z)$ as a product follows trivially from the relation between $R_{n}(z)$ and $R_{n-1}(z)$.

\subsection{The Baum-Sweet sequence}

The Baum-Sweet sequence $\mathbf{b}=\left(b_{n}\right)_{n \geqslant 0}$ is defined by $b_{0}=1, b_{2 n+1}=b_{n}, b_{4 n}=b_{n}$ and $b_{4 n+2}=0$. Its generating series

$$
\mathbf{B}(z)=\sum_{n=0}^{\infty} b_{n} z^{n}
$$

satisfies the functional equation $\mathscr{B}\left(z^{4}\right)=\mathscr{B}(z)-z \mathscr{B}\left(z^{2}\right)$.

Proposition 2.4. For any integer $n \geqslant 1$, the Padé approximant $\left[2 \cdot 4^{n} / \frac{1}{2} \cdot 4^{n}\right]_{\mathbf{B}}$ of $\mathbf{B}$, denoted by $P_{n} / Q_{n}$, can be computed recursively as follows

$$
\left\{\begin{array}{l}
Q_{n}(z)=1-z^{\frac{1}{2} \cdot 4^{n}} \\
P_{n+2}(z)=\left(1+z^{3}\right) P_{n+1}\left(z^{4}\right)-z P_{n}\left(z^{8}\right) \\
R_{n+2}(z)=\left(1+z^{3}+z^{-3}\right) R_{n+1}\left(z^{4}\right)-z^{-3} R_{n}\left(z^{16}\right)
\end{array}\right.
$$

where $R_{n}(z):=Q_{n}(z) \mathbf{B}(z)-P_{n}(z)$. We have $P_{1}(z)=1-z^{2}+z-z^{5}+z^{4}-z^{6}+z^{7}, R_{1}(z)=$ $\left(1-z^{2}\right) \mathscr{B}(z)-\left(1-z^{2}+z-z^{5}+z^{4}-z^{6}+z^{7}\right)=-z^{11}+\mathcal{O}\left(z^{12}\right)$,

$$
\begin{aligned}
P_{2}(z)=z^{31}+z^{28}-z^{27}+z^{25}-z^{24}-z^{23} & -z^{20}+z^{19}-z^{17}+z^{16}-z^{11}-z^{8}+z^{7}+z^{4}+z^{3}+z+1
\end{aligned}
$$

and $Q_{2}(z) \mathscr{B}(z)-P_{2}(z)=-z^{41}+\mathcal{O}\left(z^{44}\right)$. The polynomial $P_{n}$ has integer coefficients and degree $2 \cdot 4^{n}-1$. The order at zero of $R_{n}$ is $\frac{5}{2} \cdot 4^{n}+1$.

We omit the proof because it is the same as the proofs of the previous theorems.

The claims on $P_{n}$ are proved using the identity $Q_{n}\left(z^{4}\right)=Q_{n+1}(z)$ and the functional equation $z \mathscr{B}\left(z^{8}\right)=\mathscr{B}(z)-\left(1+z^{3}\right) \mathscr{B}\left(z^{4}\right)$. On the other hand, to prove the claims on $R_{n}$, we use another functional equation $\mathscr{B}\left(z^{16}\right)=\left(1+z^{3}+z^{6}\right) \mathscr{B}\left(z^{4}\right)-z^{3} \mathscr{B}(z)$.

\section{Padé type approximants arising from Cobham's theorem}

In the previous section, we exhibited explicit Padé approximants for the generating functions associated to some automatic sequences. This relies heavily on the fact that such functions satisfy functional equations of a particular type which can be treated by Mahler's functional method. The method introduced in [1], and made quantitative in [4], for deriving diophantine properties of automatic numbers uses a different property enjoyed by automatic sequences, namely, the occurrences of repetitive patterns. A nice way to reveal such patterns arises from the use of a classical theorem of Cobham (Theorem 3.1 below). It turns out that it also provides functional approximations for the generating functions associated to automatic sequences. In this section, we briefly described this approach and show how it is related to Padé or Padé type approximants. 


\section{Boris AdAmczewsiki and TANguy Rivoal}

\subsection{Morphisms of free monoids and Cobham's theorem}

For a finite set $\mathcal{A}$, we denote by $\mathcal{A}^{*}$ the free monoid generated by $\mathcal{A}$. The empty word $\varepsilon$ is the neutral element of $\mathcal{A}^{*}$. Let $\mathcal{A}$ and $\mathcal{B}$ be two finite sets. A map from $\mathcal{A}$ to $\mathcal{B}^{*}$ can be uniquely extended to a homomorphism between the free monoids $\mathcal{A}^{*}$ and $\mathcal{B}^{*}$. Such a homomorphism is called a morphism from $\mathcal{A}^{*}$ to $\mathcal{B}^{*}$. If there is a positive integer $k$ such that each element of $\mathcal{A}$ is mapped to a word of length $k$, then the morphism is said to be $k$-uniform or simply uniform. Similarly, a map from $\mathcal{A}$ to $\mathcal{B}$ can be uniquely extended to a homomorphism between the free monoids $\mathcal{A}^{*}$ and $\mathcal{B}^{*}$. Such a homomorphism, which is just a 1-uniform morphism, is called a coding.

A morphism $\sigma$ from $\mathcal{A}^{*}$ to itself is said to be prolongable if there exists a letter $a$ such that $\sigma(a)=a W$, where the word $W$ is such that $\sigma^{n}(W)$ is a non-empty word for every $n \geqslant 0$. In this case, the sequence of finite words $\left(\sigma^{n}(a)\right)_{n \geqslant 0}$ converges in $\mathcal{A}^{*} \cup \mathcal{A}^{\mathbb{N}}$ endowed with its usual topology (see for instance [13], Chap. 2) to an infinite word a denoted $\sigma^{\infty}(a)$. This infinite word is clearly a fixed point for $\sigma$ (extended by continuity to infinite words) and we say that $\mathbf{a}$ is generated by the morphism $\sigma$.

For instance, the Thue-Morse morphism $\tau$ defined over the alphabet $\{0,1\}$ by $\tau(0)=01$ and $\tau(1)=10$ is a 2 -uniform morphism which generates the sequence

$$
\mathbf{t}=\tau^{\infty}(0)=011010011001011010 \ldots
$$

The sequence $\mathbf{t}$, which is fixed by the 2-uniform $\tau$, is known as the Thue-Morse sequence. It is probably the most emblematic example of an automatic sequence. This example is not isolated. Indeed, uniform morphisms and automatic sequences are deeply connected, as the following result of Cobham [7] shows.

Theorem 3.1 (Cobham). A sequence is $k$-automatic if and only if it is the image under a coding of a fixed point of a $k$-uniform morphism.

\subsection{Repetitive patterns in automatic sequences}

We recall here some notation for combinatorics on words. Given a finite set $\mathcal{A}$, the length of a word $W$ on the alphabet $\mathcal{A}$ is denoted by $|W|$. For any positive integer $\ell$, we write $W^{\ell}$ for the word $W \ldots W(\ell$ times concatenation of the word $W)$. More generally, for any positive real number $x$, we denote by $W^{x}$ the word $W^{\lfloor x\rfloor} W^{\prime}$, where $W^{\prime}$ is the prefix of $W$ of length $\lceil(x-\lfloor x\rfloor)|W|\rceil$. Here, $\lfloor y\rfloor$ and $\lceil y\rceil$ denote, respectively, the integer part and the upper integer part of the real number $y$.

We recall here the main construction of [1] and [3] in the case of automatic sequences. We first observe that any infinite sequence defined on a finite alphabet has a prefix of the form $U V^{w}$, where $U$ and $V$ are two finite words, and where $w$ is a real number greater than 1 . This follows from the pigeonhole principle.

Let $\mathbf{a}=\left(a_{n}\right)_{n \geqslant 1}$ be an automatic sequence with nonnegative integer values. By Cobham's theorem, there exists a morphism $\sigma$ defined from a finite set $\mathcal{A}$ into itself, a letter $a \in \mathcal{A}$, and a coding $\varphi$ from $\mathcal{A}$ into $\mathbb{N}$ such that:

$$
\mathbf{a}=\varphi\left(\sigma^{\infty}(a)\right) .
$$

Now, let us consider a repetitive pattern of the form $U_{0} V_{0}^{w}$, with $w>1$, and which is a prefix of the sequence $\sigma^{\infty}(a)$ (such pattern exists as noted above). We then observe that the sequence a begins, for every non-negative integer $n$, with $U_{n} V_{n}^{w}$, where $U_{n}=\varphi\left(\sigma^{n}\left(U_{0}\right)\right)$ and $V_{n}=\varphi\left(\sigma^{n}\left(V_{0}\right)\right)$. Indeed, we easily check that $\varphi\left(\sigma^{n}\left(V_{0}^{w}\right)\right)=\varphi\left(\sigma^{n}\left(V_{0}\right)\right)^{w}$ since $\sigma$ is a unifrom morphism. Furthermore, $\left|U_{n}\right| /\left|V_{n}\right|=\left|U_{0}\right| /\left|V_{0}\right|$. This ensures the existence of two sequences of finite words $\left(U_{n}\right)_{n \geqslant 1},\left(V_{n}\right)_{n \geqslant 1}$, and a real number $w>1$ such that:

(i) For any $n \geqslant 1$, the word $U_{n} V_{n}^{w}$ is a prefix of the word $\mathbf{a}$;

(ii) The sequence $\left(\left|U_{n}\right| /\left|V_{n}\right|\right)_{n \geqslant 1}$ is bounded; 
(iii) The sequence $(|V n|)_{n \geqslant 1}$ is increasing.

This construction leads both to the fact that automatic numbers are either rational numbers or transcendental numbers [1] and to the irrationality measure obtained in [4].

\subsection{Padé or Padé type approximants arising from repetitive patterns}

We now observe that the previous construction can in fact be embedded in the Padé approximants method.

Let $\mathbf{A}(z)=\sum_{n=1}^{\infty} a_{n} z^{n}$ be the generating series of the automatic sequence $\mathbf{a}=\left(a_{n}\right)_{n \geqslant 1}$. Set $r_{n}=\left|U_{n}\right|$ and $s_{n}=\left|V_{n}\right|$. It is proved in [1, Lemma 1] that the combinatorial conditions $(i)$, (ii) and (iii) implies that

$$
\mathbf{A}(z)=\frac{P_{n}(z)}{Q_{n}(z)}+\mathcal{O}\left(z^{r_{n}+\left\lceil w s_{n}\right\rceil+1}\right)
$$

where $Q_{n}(z)=1-z^{s_{n}}$ and

$$
P_{n}(z)=\left(1-z^{s_{n}}\right) \sum_{k=1}^{r_{n}} a_{k} x^{k}+x^{r_{n}} \sum_{k=1}^{s_{n}} a_{r_{n}+k} x^{k} .
$$

We can thus rewrite (5) as

$$
Q_{n}(z) \mathbf{A}(z)-P_{n}(z)=\mathcal{O}\left(z^{r_{n}+\left\lceil w s_{n}\right\rceil+1}\right) .
$$

Since $\operatorname{deg}\left(P_{n}\right)=r_{n}+s_{n}$ and $\operatorname{deg}\left(Q_{n}\right)=s_{n}$, the order at zero of $Q_{n}(z) \mathbf{A}(z)-P_{n}(z)$ indicates that $P_{n} / Q_{n}$ is the Padé approximant $\left[r_{n}+s_{n} / s_{n}\right]_{\mathbf{A}}$ of $\mathbf{A}$ if $w \geqslant 2$. If $1<w<2$ (a case which happens), then $P_{n} / Q_{n}$ is just a Padé type approximant. Actually, we will see in 4.3 that this case happens for the generating function of the Thue-Morse, Rudin-Shapiro, paperfolding and BaumSweet sequences, while this method provides "real" Padé approximants for the generating function of Thue-Morse like sequences associated with a parameter $k>5$.

In [4], it is shown that, when the sequence $\mathbf{a}$ is not eventually periodic, the functional approximation (5) always provides a nontrivial upper bound for the irrationality exponent of the automatic numbers $\mathbf{A}(1 / b)$, where $b \geqslant 2$ is an integer. Thus, this approach turns out to be $a$ posteriori very close in spirit to the construction of Padé approximants made for our special sequences in Section 2. It is worth mentioning that the irrationality measure that can be derived from such construction essentially depends on the choice of the initial repetitive pattern $U_{0} V_{0}^{w}$ and especially on the value of the ratio $\left|U_{0} V_{0}^{w}\right| /\left|U_{0} V_{0}\right|$ (which has to be as large as possible). Such a pattern has no reason to be unique and actually there are always infinitely many of them. The choice of the initial pattern thus becomes very important when studying specific examples. This point should become more transparent in Subsection 4.3.

\section{Irrationality measures for numbers associated with famous automatic sequences}

We are now interested in finding upper bounds for the irrationality exponent of various famous automatic numbers, namely those associated with Thue-Morse like sequences, Rudin-Shapiro, paperfolding and Baum-Sweet sequences. For all these numbers, we will obtain irrationality measures by two different ways. The first involves the Padé approximants provided in Section 2, while the

second builds on the approach outlined in Section 3. Both methods will then be compared in Subsection 4.4.

\subsection{An approximation lemma}

We first prove the following result from which we will derive all our irrationality measures. 


\section{Boris AdAmczeWsin and TANGuy Rivoal}

Lemma 4.1. Let $\xi, \delta, \rho$ and $\theta$ be real numbers such that $0<\delta \leqslant \rho$ and $\theta \geqslant 1$. Let us assume that there exists a sequence $\left(p_{n} / q_{n}\right)_{n \geqslant 1}$ of rational numbers and some positive constants $c_{0}, c_{1}$ and $c_{2}$ such that

(i) $q_{n}<q_{n+1} \leqslant c_{0} q_{n}^{\theta}$;

(ii) $\frac{c_{1}}{q_{n}^{1+\rho}} \leqslant\left|\xi-\frac{p_{n}}{q_{n}}\right| \leqslant \frac{c_{2}}{q_{n}^{1+\delta}}$.

Then we have that

$$
\mu(\xi) \leqslant(1+\rho) \theta / \delta .
$$

Furthermore, if we also assume that

(iii) for any $n \gg 1, p_{n}$ and $q_{n}$ are coprime,

then we have $\theta \geqslant \delta$ and

$$
\mu(\xi) \leqslant \max (1+\rho, 1+\theta / \delta) .
$$

Remark 3 . The hypothesis of the lemma ensure that we always have $1+\theta / \delta \leqslant(1+\rho) \theta / \delta$. Since $\theta \geqslant \delta$ when $(i),(i i)$ and $(i i i)$ hold, we therefore have

$$
\max (1+\rho, 1+\theta / \delta) \leqslant(1+\rho) \theta / \delta,
$$

hence (7) is an improvement on (6). We are not able to prove coprimality in the examples worked out in the next sections but it is likely that it could be done for some of these.

Proof. Let $p / q$ be a rational number whose denominator is large enough. Then, there exists a unique integer $n=n(q) \geqslant 2$ such that $q_{n-1}<\left(2 c_{2} q\right)^{1 / \delta} \leqslant q_{n}$. By the triangle inequality, we have that

$$
\left|\xi-\frac{p}{q}\right| \geqslant\left|\frac{p}{q}-\frac{p_{n}}{q_{n}}\right|-\left|\xi-\frac{p_{n}}{q_{n}}\right| \text {. }
$$

If $p / q \neq p_{n} / q_{n}$, then $\left|\frac{p}{q}-\frac{p_{n}}{q_{n}}\right| \geqslant \frac{1}{q q_{n}}$ and we deduce from $(i i)$ and $\left(2 c_{2} q\right)^{1 / \delta} \leqslant q_{n}$ that

$$
\left|\xi-\frac{p}{q}\right| \geqslant \frac{1}{2 q q_{n}}
$$

By $(i)$, we have $q_{n} \leqslant c_{0} q_{n-1}^{\theta}<c_{0}\left(2 c_{2}\right)^{\theta / \delta} q^{\theta / \delta}$ and therefore

$$
\left|\xi-\frac{p}{q}\right| \geqslant \frac{1}{c_{3} q^{1+\theta / \delta}}
$$

where $c_{3}=2 c_{0}\left(2 c_{2}\right)^{\theta / \delta}$.

On the other hand, if $p / q=p_{n} / q_{n}$, we get that

$$
\left|\xi-\frac{p}{q}\right|=\left|\xi-\frac{p_{n}}{q_{n}}\right| \geqslant \frac{c_{1}}{q_{n}^{1+\rho}} \geqslant \frac{1}{c_{4} q^{(1+\rho) \theta / \delta}},
$$

where $c_{4}=c_{1}^{-1} c_{0}^{1+\rho}\left(2 c_{2}\right)^{(1+\rho) \theta / \delta}$. This concludes the proof of (6) because the assumptions $0<\delta \leqslant \rho$ and $\theta \geqslant 1$ imply that $1+\theta / \delta \leqslant(1+\rho) \theta / \delta$.

We now turn our attention to the case where assumption (iii) holds. We initially use the same decomposition into two cases as in the above proof. We note that without loss of generality we can assume that $p / q$ is reduced. Hence, in the second case " $p / q=p_{n} / q_{n}$ ", we deduce that $q=q_{n}$ and hence that $\left|\xi-\frac{p}{q}\right| \geqslant c_{1} q^{-1-\rho}$. Remember that this holds under the condition $q_{n-1}<\left(2 c_{2} q\right)^{1 / \delta} \leqslant q_{n}$, which together with $q=q_{n}$ implies (see (8)) that

$$
c_{1}^{-1} q^{1+\rho} \leqslant c_{4} q^{(1+\rho) \theta / \delta} .
$$




\section{IRRATIONALITY MEASURES FOR SOME AUTOMATIC REAL NUMBERS}

There are now two cases : either there exist infinitely many $q$ such that $q=q_{n}$ and $q_{n-1}<\left(2 c_{2} q\right)^{1 / \delta} \leqslant$ $q_{n}$ simultaneously (for the unique $n$ possible), or not.

In the first case, it follows that (9) holds for infinitely many $q$ and we get the measure $\mu(\xi) \leqslant$ $\max (1+\rho, 1+\theta / \delta)$ by comparing the cases " $p / q \neq p_{n} / q_{n}$ " and " $p_{n} / q_{n}=p / q$ ". Also, passing to the limit $q \rightarrow+\infty$ in (9) (along a suitable subsequence) implies that $1+\rho \leqslant(1+\rho) \theta / \delta$ and since we always have $1+\theta / \delta \leqslant(1+\rho) \theta / \delta$, we deduce that $\max (1+\rho, 1+\theta / \delta) \leqslant(1+\rho) \theta / \delta$. In other words, (7) improves on (6). Note that $1+\rho \leqslant(1+\rho) \theta / \delta$ implies that $\theta \geqslant \delta$.

In the second case, only the case " $p / q \neq p_{n} / q_{n}$ " contributes to the computation of the measure of $\xi$. This gives the upper bound $\mu(\xi) \leqslant 1+\theta / \delta$. We note that this bound implies that $\theta \geqslant \delta$ because $\mu(\alpha) \geqslant 2$ for any irrational number $\alpha$. In turn, this implies the last inequality in the following chain:

$$
\mu(\xi) \leqslant 1+\theta / \delta \leqslant \max (1+\rho, 1+\theta / \delta) \leqslant(1+\rho) \theta / \delta .
$$

Hence in this case, (7) holds and improves on (6).

In summary, the additional assumption that $p_{n}$ and $q_{n}$ are coprime for all $n \gg 1$ implies that $\theta \geqslant \delta$ and provides the improved upper bound $\mu(\xi) \leqslant \max (1+\rho, 1+\theta / \delta)$, as expected.

\subsection{Measures deduced from the Padé approximants obtained in Section 2}

We use here the various Padé approximants constructed in Section 2 to derive irrationality measures for the numbers $\mathbf{T}_{k}(1 / b), \mathbf{R}(1 / b), \mathbf{F}(1 / b)$ and $\mathbf{B}(1 / b)$, where $b \geqslant 2$ is an integer.

The two numbers $\mathbf{T}_{k}(1 / b)$ and $\mathbf{B}(1 / b)$ are automatic numbers for any $b \geqslant 2$ because $s_{n}, b_{n} \in$ $\{0,1\}$. But strictly speaking, $\mathbf{R}(1 / b)$ and $\mathbf{F}(1 / b)$ are not automatic numbers because $r_{n}, f_{n} \in$ $\{-1,1\}$. However, the sequences defined by $\widehat{r}:=\left(r_{n}+1\right) / 2$ and $\widehat{f}_{n}:=\left(f_{n}+1\right) / 2$ take their values in $\{0,1\}$ and are still automatic. Thus

$$
\widehat{\mathbf{R}}(1 / b):=\frac{1}{2} \sum_{n=0}^{\infty} \frac{r_{n}+1}{b^{n}} \text { and } \widehat{\mathbf{F}}(1 / b):=\frac{1}{2} \sum_{n=0}^{\infty} \frac{f_{n}+1}{b^{n}}
$$

are automatic numbers in base $b \geqslant 2$. Obviously, $\widehat{\mathbf{R}}(1 / b)$ and $\widehat{\mathbf{F}}(1 / b)$ have the same irrationality as $\mathbf{R}(1 / b)$ and $\mathbf{F}(1 / b)$ respectively. Hence, we can abuse terminology and call all these numbers "automatic".

Let us fix $b \geqslant 2$. The implicit constants in the $\gg$ symbols below are effective and could be explicitly computed if needed.

4.2.1 The Thue-Morse sequence. In the Padé approximations obtained in Theorem 2.1 for $k=2$, we set $x=1 / b$. We define $q_{n}=b^{3.2^{n}-1} Q_{n}(1 / b)$ and $p_{n}=b^{3.2^{n}-1} P_{n}(1 / b)$, which are integers, so that

$$
\mathbf{T}_{2}(1 / b)-\frac{p_{n}}{q_{n}}=\frac{b^{3.2^{n}-1}}{q_{n}} R_{n}(1 / b) .
$$

We recall that $\sigma_{2}(z)=-(1-z)^{2}$. Consequently,

$$
R_{n}(z)=z^{2^{n}-1} R_{0}\left(z^{2^{n}}\right) \prod_{j=1}^{n}\left(1-z^{2^{j}}\right)
$$

and $R_{0}(z)=-z^{5}+\mathcal{O}\left(z^{6}\right)$. This last assertion implies that for $n \gg 1, R_{0}\left(b^{-2^{n}}\right) \neq 0$ and even $R_{0}\left(b^{-2^{n}}\right) \sim-b^{-5 \cdot 2^{n}}$, where the implicit constants depend on $b$. Thus $R_{n}(1 / b) \neq 0$ for $n \gg 1$ and since the product in (10) converges (to $A$ say), we obtain

$$
R_{n}(1 / b) \sim \frac{A}{b^{6 \cdot 2^{n}-1}} \quad, n \rightarrow+\infty .
$$




\section{Boris AdAmczeWsin and TANGuy Rivoal}

Furthermore, the expression for $Q_{n}(x)$ implies that $q_{n} \sim b^{3.2^{n}-1}$ and hence

$$
R_{n}(1 / b) \sim \frac{A_{1}}{q_{n}^{2}} \quad, n \rightarrow+\infty
$$

for some constant $A_{1} \neq 0$.

Hence, we are now in position to apply Lemma 4.1 to the approximations

$$
\mathbf{T}_{2}(1 / b)-\frac{p_{n}}{q_{n}} \sim \frac{A_{1}}{q_{n}^{2}},
$$

with $\theta=2$ and $\rho=\delta=1$. We get $\mu\left(\mathbf{T}_{2}(1 / b)\right) \leqslant 4$.

Remark 4. Eq. (11) suggests that $\left(p_{n} / q_{n}\right)_{n}$ is a subsequence of the convergents of $\mathbf{T}_{2}(1 / b)$ : we will prove this fact in Section 5 by showing that $\left|A_{1}\right|<1 / 2$.

Furthermore, it seems that $p_{n}$ and $q_{n}$ are always coprime, which would imply the even stronger fact that $\left(p_{n}\right)_{n}$ and $\left(q_{n}\right)_{n}$ are subsequences of the numerators and denominators, respectively, of the convergents of $\mathbf{T}_{2}(1 / b)$. We do not know how to prove coprimality.

4.2.2 The Thue-Morse like sequence, $k \geqslant 4$. Repeating Mutadis mutandis the previous subsection, we obtain that $\mu\left(\mathbf{T}_{k}(1 / b)\right) \leqslant k(k+3) / 3$.

Indeed, we have the approximation

$$
\mathbf{T}_{k}(1 / b)-\frac{p_{n}}{q_{n}} \sim \frac{c_{0}}{q_{n}^{1+3 / k}},
$$

where $q_{n}=b^{k^{n+1}-1} Q_{n}(1 / b) \in \mathbb{Z}$ and $p_{n}=b^{k^{n+1}-1} P_{n}(1 / b) \in \mathbb{Z}$. We have $q_{n} \sim b^{k^{n+1}-1}$ and thus we can apply Lemma 4.1 with $\theta=k$ and $\rho=\delta=3 / k$.

Remark 5. We observe that the upper bound obtained for $\mu\left(\mathbf{T}_{k}(1 / b)\right)$ is not very good (and, indeed, will be improved later) because our Padé approximants are far from diagonal (i.e., such that the degrees of the numerator and denominator are equal). Here, we have $\operatorname{deg}\left(P_{n}\right) / \operatorname{deg}\left(Q_{n}\right) \sim k$ as $k$ or $n \rightarrow+\infty$.

4.2.3 The Rudin-Shapiro sequence. In the Padé approximations obtained in Theorem 2.2, we set $x=1 / b$ and define $q_{n}=b^{\frac{5}{2} \cdot 2^{n}-1} Q_{n}(1 / b)$ and $p_{n}=b^{\frac{5}{2} \cdot 2^{n}-1} P_{n}(1 / b)$, which are integers, so that

$$
\mathbf{R}(1 / b)-\frac{p_{n}}{q_{n}}=\frac{b^{\frac{5}{2} \cdot 2^{n}-1}}{q_{n}} R_{n}(1 / b) \text {. }
$$

We have $q_{n} \sim b^{\frac{5}{2} \cdot 2^{n}-1}$. It is possible to prove that $R_{n}(1 / b) \sim c_{0} b^{-\frac{7}{2} \cdot 2^{n}}$ for some constant $c_{0} \neq 0$ : we will not give the proof of this property because the measure thus obtained is weaker than that obtained in 4.3 by simpler means. Admitting this fact, we get that

$$
\mathbf{R}(1 / b)-\frac{p_{n}}{q_{n}} \sim \frac{c_{1}}{q_{n}^{1+2 / 5}}
$$

and we can apply Lemma 4.1 with $\theta=2, \rho=\delta=2 / 5$ to get $\mu(\mathbf{R}(1 / b)) \leqslant 7$.

4.2.4 The paperfolding sequence. In the Padé approximations obtained in Theorem 2.3, we set $x=1 / b$ and define $q_{n}=b^{3.2^{n}} Q_{n}(1 / b)$ and $p_{n}=b^{3.2^{n}} P_{n}(1 / b)$, which are integers, so that

$$
\mathbf{F}(1 / b)-\frac{p_{n}}{q_{n}}=\frac{b^{3.2^{n}}}{q_{n}} R_{n}(1 / b) .
$$


We know that

$$
R_{n}(z)=z^{2^{n-1}-1} R_{1}\left(z^{2^{n-1}}\right) \prod_{j=1}^{n-1}\left(1-z^{2^{j}}\right)
$$

and $R_{0}(z)=2 z^{9}+\mathcal{O}\left(z^{10}\right)$. This implies that for $n \gg 1, R_{1}\left(b^{-2^{n-1}}\right) \neq 0$ and even $R_{1}\left(b^{-2^{n-1}}\right) \sim$ $2 b^{-9 \cdot 2^{n-1}}$, where the implicit constants depend on $b$. Thus $R_{n}(1 / b) \neq 0$ for $n \gg 1$ and since the product in (12) converges (to $B$ say), we obtain

$$
R_{n}(1 / b) \sim \frac{2 B}{b^{5 \cdot 2^{n}}} \quad, n \rightarrow+\infty
$$

Furthermore, the expression for $Q_{n}(x)$ implies that $q_{n} \sim c_{0} b^{3.2^{n}}$ for some $c_{0} \neq 0$, hence

$$
R_{n}(1 / b) \sim \frac{B_{1}}{q_{n}^{5 / 3}} \quad, n \rightarrow+\infty
$$

for some constant $B_{1} \neq 0$.

We can now apply Lemma 4.1 to the approximations

$$
\mathbf{F}(1 / b)-\frac{p_{n}}{q_{n}} \sim \frac{B_{2}}{q_{n}^{1+2 / 3}},
$$

with $\theta=2$ and $\rho=\delta=2 / 3$ and finally we get $\mu(\mathbf{F}(1 / b)) \leqslant 5$.

4.2.5 The Baum-Sweet sequence. In the Padé approximations obtained in Theorem 2.2, we set $x=1 / b$ and define $q_{n}=b^{2 \cdot 4^{n}-1} Q_{n}(1 / b) \in \mathbb{Z}$ and $p_{n}=b^{2 \cdot 4^{n}-1} P_{n}(1 / b) \in \mathbb{Z}$, which are integers, so that

$$
\mathbf{R}(1 / b)-\frac{p_{n}}{q_{n}}=\frac{b^{2 \cdot 4^{n}-1}}{q_{n}} R_{n}(1 / b) .
$$

We have $q_{n} \sim b^{2 \cdot 4^{n}-1}$. It is possible to prove that $R_{n}(1 / b) \sim c_{0} b^{-\frac{5}{2} \cdot 4^{n}}$ for some constant $c_{0} \neq 0$. As for the Rudin-Shapiro, we will not give the proof of this property because the measure obtained is weaker than that obtained in 4.3. Admitting this fact, we get that

$$
\mathbf{R}(1 / b)-\frac{p_{n}}{q_{n}} \sim \frac{c_{1}}{q_{n}^{1+1 / 4}}
$$

and we can apply Lemma 4.1 with $\theta=4, \rho=\delta=1 / 4$ to get $\mu(\mathbf{R}(1 / b)) \leqslant 20$.

\subsection{Measures arising from Cobham's theorem}

In this section, we use the approach outlined in Section 3 to derive upper bounds for the irrationality exponent of the automatic numbers considered in the previous subsection.

In what follows, an expression of the form $p / q=0 . U V^{\infty}$ will mean that the rational number $p / q$ has an eventually periodic $b$-ary expansion with preperiod $U$ and period $V$.

4.3.1 The Thue-Morse like sequences. Let $k \geqslant 2$ be an integer. It is easy to prove that the sequence $\mathbf{t}_{k}$ is the fixed point beginning with 0 of the binary morphism $\tau_{k}$ defined by

$$
\tau_{k}(0)=010^{k-2} \text { and } \tau_{k}(1)=101^{k-2} .
$$

- If $k=2$, we observe that $\mathbf{t}_{k}$ begins with $U_{n} V_{n}^{w}$, where $U_{n}$ denotes the empty word, $V_{n}=\tau_{k}^{n}(011)$ and $w=1+2 / 3$. Set $\delta:=\left|U_{n} V_{n}^{w}\right| /\left|U_{n} V_{n}\right|-1=2 / 3$. Let us consider the positive integer $q_{n}=b^{3 \cdot 2^{n}}-1$. Then there exists a positive integer $p_{n}$ such that

$$
\frac{p_{n}}{q_{n}}:=0 . U_{n} V_{n}^{\infty} .
$$




\section{Boris AdAmczewsiki and TANguy Rivoal}

It follows from a simple computation (see [4]) that

$$
\frac{1}{q_{n}^{1+\delta}} \ll\left|\mathbf{T}_{2}(1 / b)-\frac{p_{n}}{q_{n}}\right| \ll \frac{1}{q_{n}^{1+\delta}} \text { and } q_{n}<q_{n+1} \ll q_{n}^{2},
$$

where the constants implied by the symbol $\ll$ do not depend on $n$. We then deduce from Lemma 4.1 that

$$
\mu\left(\mathbf{T}_{2}(1 / b)\right) \leqslant 5
$$

a result already proved in [4].

- If $k=3$, we recall that $\mathbf{T}_{3}(1 / b)$ is rational. Consequently, our method does not apply in this case.

- If $4 \leqslant k \leqslant 5$, we observe that $\mathbf{t}_{k}$ begins with $U_{n} V_{n}^{w}$, where $U_{n}$ denotes the empty word, $V_{n}=\tau_{k}^{n}\left(010^{k-3}\right)$ and $w=1+3 /(k-1)$. Set $\delta:=\left|U_{n} V_{n}^{w}\right| /\left|U_{n} V_{n}\right|-1=3 /(k-1)$. Let us consider the positive integer $q_{n}=b^{(k-1) \cdot k^{n}}-1$. Then there exists a positive integer $p_{n}$ such that

$$
\frac{p_{n}}{q_{n}}:=0 . U_{n} V_{n}^{\infty} .
$$

We deduce from a computation similar to the one in [4] for the cae $k=2$ that

$$
\frac{1}{q_{n}^{1+\delta}} \ll\left|\mathbf{T}_{k}(1 / b)-\frac{p_{n}}{q_{n}}\right| \ll \frac{1}{q_{n}^{1+\delta}} \text { and } q_{n}<q_{n+1} \ll q_{n}^{k},
$$

where the constants implied by the symbol $\ll$ do not depend on $n$. Actually, it is sufficient to observe that $p_{n} / q_{n}$ and $\mathbf{T}_{k}(1 / b)$ have the same first $(k+2) \cdot k^{n}$ digits in their $b$-ary expansion, while their $\left((k+2) \cdot k^{n}+1\right)$-th digits are different. We then deduce from Lemma 4.1 that

$$
\mu\left(\mathbf{T}_{k}(1 / b)\right) \leqslant \frac{k(k+2)}{3} .
$$

- If $k>5$, we note that $\mathbf{t}_{k}$ begins with $U_{n} V_{n}^{w}$, where $U_{n}=\sigma_{k}^{n}(01)$ denotes the empty word, $V_{n}=\tau_{k}^{n}(0)$ and $w=k-2$. Set $\delta:=\left|U_{n} V_{n}^{w}\right| /\left|U_{n} V_{n}\right|-1=(k-3) / 3$. Then, setting $q_{n}=b^{2 \cdot k^{n}}\left(b^{k^{n}}-1\right)$, there exists a positive integer $p_{n}$ such that

$$
\frac{p_{n}}{q_{n}}=0 . U_{n} V_{n}^{\infty}
$$

We can show as previously that

$$
\frac{1}{q_{n}^{1+\delta}} \ll\left|\mathbf{T}_{k}(1 / b)-\frac{p_{n}}{q_{n}}\right| \ll \frac{1}{q_{n}^{1+\delta}} \text { and } q_{n}<q_{n+1} \ll q_{n}^{k},
$$

where the constants implied by the symbol $\ll$ do not depend on $n$. We then deduce from Lemma 4.1 that

$$
\mu\left(\mathbf{T}_{k}(1 / b)\right) \leqslant \frac{k^{2}}{k-3} .
$$

In all case, this provides a stronger irrationality measure than that obtained via exact Padé approximants. Furthermore, for every $k>6$, this method provides the non-trivial lower bound $1+\delta=k / 3$ for the irrationality exponent of $\left.\mathbf{T}_{k}(1 / b)\right)$. In particular, a simple computation shows that if we could prove $p_{n}$ and $q_{n}$ to be coprime (or that their ged is small enough), we would obtain the exact value of the irrationality exponent of $\mathbf{T}_{k}(1 / b)$ for every $k \geqslant 15$. Namely, Lemma 4.1 would ensure that $\mu\left(\mathbf{T}_{k}(1 / b)\right)=k / 3$.

4.3.2 The Rudin-Shapiro sequence. Let $\rho$ be a morphism defined from $\{a, b, c, d\}^{*}$ into itself by: $\rho(a)=a b, \rho(b)=a c, \rho(c)=d b$ and $\rho(d)=d c$. Let

$$
\mathbf{u}_{\mathbf{1}}=a b a c a b d b \ldots
$$




\section{IRRATIONALITY MEASURES FOR SOME AUTOMATIC REAL NUMBERS}

be the fixed point of $\rho$ beginning with $a$ and let $\varphi_{1}$ be the morphism defined from $\{a, b, c, d\}^{*}$ to $\{0,1\}^{*}$ by: $\varphi_{1}(a)=\varphi_{1}(b)=0$ and $\varphi_{1}(c)=\varphi_{1}(d)=1$. It is well-known that the Rudin-Shapiro sequence $\mathbf{r}$ can be obtained as

$$
\mathbf{r}=\varphi_{1}\left(\mathbf{u}_{1}\right)
$$

We thus observe that $\mathbf{r}$ begins with $U_{n} V_{n}^{w}$ where $U_{n}$ denotes the empty word, $V_{n}=\varphi_{1}\left(\rho^{n}(a b)\right)$ and $w=3 / 2$. Set $\delta:=\left|U_{n} V_{n}^{w}\right| /\left|U_{n} V_{n}\right|-1=1 / 2$. Then, setting $q_{n}=b^{2^{n+1}}-1$, there exists a positive integer $p_{n}$ such that

$$
\frac{p_{n}}{q_{n}}=0 . U_{n} V_{n}^{\infty} .
$$

We then deduce from the definition of $\rho$ and $\varphi$ that $p_{n} / q_{n}$ and $\mathbf{R}(1 / b)$ have the same first $3 \cdot 2^{n}$ digits in their $b$-ary expansion, while their $\left(3 \cdot 2^{n}\right)+1$-th digits are different. Moreover, $\mathbf{R}(1 / 2)$ does not have arbitrarily large blocks of 1's in its binary expansion. Consequently, we get that

$$
\frac{1}{q_{n}^{1+\delta}} \ll\left|\mathbf{R}(1 / b)-\frac{p_{n}}{q_{n}}\right| \ll \frac{1}{q_{n}^{1+\delta}} \text { and } q_{n}<q_{n+1} \ll q_{n}^{2}
$$

where the constants implied by the symbol $\ll$ do not depend on $n$. By Lemma 4.1, we get that

$$
\mu(\mathbf{R}(1 / b)) \leqslant 6 .
$$

Though the rational approximations under consideration are different, this approach provides a better irrationality measure than the one obtained using exact Padé approximants.

4.3.3 The paperfolding sequence. Let $\phi$ be a morphism defined from $\{a, b, c, d\}^{*}$ into itself by: $\phi(a)=a b, \phi(b)=c b, \phi(c)=a d$ and $\phi(d)=c d$. Let

$$
\mathbf{u}_{\mathbf{2}}=a b c b a d c b a b \ldots
$$

be the fixed point of $\phi$ beginning with $a$ and let $\varphi_{2}$ be the morphism defined from $\{a, b, c, d\}^{*}$ to $\{0,1\}^{*}$ by: $\varphi_{2}(a)=\varphi_{2}(b)=1$ and $\varphi_{2}(c)=\varphi_{2}(d)=0$. It is well-known that the paperfolding sequence $\mathbf{f}$ can be obtained as

$$
\mathbf{f}=\varphi_{2}\left(\mathbf{u}_{\mathbf{2}}\right) .
$$

It can be shown that $\mathbf{p}$ begins with $U_{n} V_{n}^{w}$ where $U_{n}=\varphi_{2}\left(\phi^{n}(a b)\right)$ denotes the empty word, $V_{n}=$ $\varphi_{2}\left(\phi^{n}(\operatorname{cbad})\right)$ and $w=1+7 / 8$. Set $\delta:=\left|U_{n} V_{n}^{w}\right| /\left|U_{n} V_{n}\right|-1=15 / 24$. Then, setting $q_{n}=b^{2^{n+1}}\left(b^{\cdot 2^{n+2}}-\right.$ $1)$, there exists a positive integer $p_{n}$ such that

$$
\frac{p_{n}}{q_{n}}=0 . U_{n} V_{n}^{\infty}
$$

Setting $\rho=2 / 3$, we can show that

$$
\frac{1}{q_{n}^{1+\rho}} \ll\left|\mathbf{F}(1 / b)-\frac{p_{n}}{q_{n}}\right| \ll \frac{1}{q_{n}^{1+\delta}} \text { and } q_{n}<q_{n+1} \ll q_{n}^{2}
$$

where the constants implied by the symbol « do not depend on $n$ (we omit the details but there is no hidden catch). We then deduce from Lemma 4.1 that

$$
\mu(\mathbf{F}(1 / b)) \leqslant 5+1 / 3 .
$$

In fact, a more careful analysis lets us reduce the value $5+1 / 3$ to 5 . With such a refinement, irrationality measures arising from periodic patterns turn out to match the value 5 obtained via exact Padé approximants. 


\section{Boris AdAmczewsiki and TANguy Rivoal}

4.3.4 The Baum-Sweet sequence. Let $\beta$ be a morphism defined from $\{a, b, c, d\}\}^{*}$ into itself by $\beta(a)=a b, \beta(b)=c b, \beta(c)=b d$ and $\beta(d)=d d$. Let

$$
\mathbf{u}_{3}=a b c b b d c b c b d d \ldots
$$

be the fixed point of $\beta$ beginning with $a$. It is well-known that the paperfolding sequence $\mathbf{b}$ can be obtained as

$$
\mathbf{b}=\varphi_{2}\left(\mathbf{u}_{2}\right) .
$$

We then observe that $\mathbf{b}$ begins with $U_{n} V_{n}^{w}$ where $U_{n}=\varphi_{2}\left(\beta^{n}(a)\right)$ denotes the empty word, $V_{n}=$ $\varphi_{2}\left(\beta^{n}(b c b b d c)\right)$ and $w=3 / 2$. Set $\delta:=\left|U_{n} V_{n}^{w}\right| /\left|U_{n} V_{n}\right|-1=3 / 7$. Then setting $q_{n}=b^{2^{n}}\left(b^{3 \cdot 2^{n+1}}-1\right)$, there exists a positive integer $p_{n}$ such that

$$
\frac{p_{n}}{q_{n}}=0 . U_{n} V_{n}^{\infty}
$$

We observe now that $p_{n} / q_{n}$ and $\mathbf{B}(1 / b)$ have the same first $10 \cdot 2^{n}$ digits in their $b$-ary expansion, while their $\left(10 \cdot 2^{n}+1\right)$-th digits are different. Consequently, we obtain that

$$
\frac{1}{q_{n}^{1+\delta}} \ll\left|\mathbf{B}(1 / b)-\frac{p_{n}}{q_{n}}\right| \ll \frac{1}{q_{n}^{1+\delta}} \text { and } q_{n}<q_{n+1} \ll q_{n}^{2},
$$

where the constants implied by the symbol $\ll$ do not depend on $n$. We then deduce from Lemma 4.1 that

$$
\mu(\mathbf{B}(1 / b)) \leqslant 6+2 / 3 .
$$

We observe that this irrationality measure is much stronger that the one obtained using exact Padé approximants.

\subsection{Comparison of the approaches}

We now compare the irrationality measures given by these two Padé (type) approximants. The following theorem collects the best irrationality measures obtained in the two previous subsections. We observe that the Padé approximants constructed in Section 2 provide better irrationality measures only in the case of the Thue-Morse sequence and the paperfolding sequence, while Padé type approximants arising from Cobham's theorem give better irrationality measures in all other cases. However, it was difficult to predict a priori which approach would yield better results.

TheOrem 4.2. Let $b \geqslant 2$ be an integer.

(i) Thue-Morse. For any $(p, q) \in \mathbb{Z} \times \mathbb{N}^{\times}$, where $p, q$ are coprime,

$$
\left|\mathbf{T}_{2}(1 / b)-\frac{p}{q}\right| \gg \frac{1}{q^{4}}
$$

(ii) Thue-Morse like, $k=4,5$. For any $(p, q) \in \mathbb{Z} \times \mathbb{N}^{\times}$, where $p, q$ are coprime,

$$
\left|\mathbf{T}_{k}(1 / b)-\frac{p}{q}\right| \gg \frac{1}{q^{k(k+2) / 3}} .
$$

(iii) Thue-Morse like, $k \geqslant 6$. For any $(p, q) \in \mathbb{Z} \times \mathbb{N}^{\times}$, where $p, q$ are coprime,

$$
\left|\mathbf{T}_{k}(1 / b)-\frac{p}{q}\right| \gg \frac{1}{q^{k^{2} /(k-3)}}
$$

(iv) Rudin-Shapiro. For any $(p, q) \in \mathbb{Z} \times \mathbb{N}^{\times}$, where $p, q$ are coprime,

$$
\left|\mathbf{R}(1 / b)-\frac{p}{q}\right| \gg \frac{1}{q^{6}}
$$




\section{IRRATIONALITY MEASURES FOR SOME AUTOMATIC REAL NUMBERS}

(v) Paper folding. For any $(p, q) \in \mathbb{Z} \times \mathbb{N}^{\times}$, where $p, q$ are coprime,

$$
\left|\mathbf{F}(1 / b)-\frac{p}{q}\right| \gg \frac{1}{q^{5}}
$$

(vi) Baum-Sweet. For any $(p, q) \in \mathbb{Z} \times \mathbb{N}^{\times}$, where $p, q$ are coprime,

$$
\left|\mathbf{B}(1 / b)-\frac{p}{q}\right| \gg \frac{1}{q^{6+2 / 3}} .
$$

Remarks 6. (a) We do not claim that the above irrationality measures are optimal. In fact, it is likely they are not, particularly for the Thue-Morse sequence (see the remark at the end of subsection 4.2.1).

(b) The same techniques allows one to obtain irrationality measures for similar numbers where we replace $1 / b$ by $a / b$. Furthermore, the non diagonal Padé approximants used in this paper might be useful to study the approximation of numbers like $\mathbf{T}_{k}(a / b)$ by fractions of the form $u / b^{m}$ (see [15] for a related study in the case of values of the logarithm).

\section{On the convergents of the numbers $\mathbf{T}_{2}(1 / b)$}

In this section, we prove the following theorem, which was announced earlier in the paper. As far as we know, this is the first result concerning the continued fractions of the numbers $\mathbf{T}_{2}(1 / b)$.

Theorem 5.1. Let $P_{n}(X)$ and $Q_{n}(X)$ be the polynomials of $\mathbb{Z}[X]$ of degree at most $3 \cdot 2^{n}-1$ defined in Proposition 2.1 for $k=2$ by $P_{0}(X)=1+X-X^{2}$ and

$$
\begin{aligned}
Q_{n}(X) & =(1-X)\left(1+X^{2^{n}}\right), \\
P_{n+1}(X) & =\frac{1}{1-X^{2}} Q_{n+1}(X)+\frac{X(1-X)}{1+X} P_{n}\left(X^{2}\right) .
\end{aligned}
$$

For any integer $b \geqslant 2$, we also define the integers $q_{n}(b)=b^{3 \cdot 2^{n}-1} Q_{n}(1 / b)=b^{2 \cdot 2^{n}-2}(b-1)\left(b^{2^{n}}+1\right)$ and $p_{n}(b)=b^{3 \cdot 2^{n}-1} P_{n}(1 / b)$.

Then for any integer $n \geqslant 1$, the rational number $\frac{p_{n}(b)}{q_{n}(b)}$ is a convergent of the number $\mathbf{T}_{2}(1 / b)$.

Proof. From now on, $b \geqslant 2$ is fixed and we set $p_{n}=p_{n}(b)$ and $q_{n}=q_{n}(b)$. We have

$$
\mathbf{T}_{2}(1 / b)-\frac{p_{n}}{q_{n}}=\frac{b^{3 \cdot 2^{n}-1}}{q_{n}} R_{n}(1 / b)
$$

where $R_{n}(X)=Q_{n}(X) \mathbf{T}_{2}(X)-P_{n}(X)$. By the classical theory of continued fractions, it will be enough to prove that

$$
\left|\frac{b^{3 \cdot 2^{n}-1}}{q_{n}} R_{n}(1 / b)\right|<\frac{1}{2 q_{n}^{2}} .
$$

To do this, we will work with the quantity $D_{n}:=b^{3 \cdot 2^{n}-1} q_{n} R_{n}(1 / b)$ and show that its absolute value is $<1 / 2$. Using the expression given for $R_{n}$ in Proposition 2.1, we find that

$$
D_{n}=b^{6 \cdot 2^{n}-2}\left(1-\frac{1}{b}\right)\left(1+\frac{1}{b^{2^{n}}}\right) \frac{R_{0}\left(b^{-2^{n}}\right)}{b^{2^{n}-1}} \prod_{j=1}^{n}\left(1-\frac{1}{b^{2^{j}}}\right) .
$$

We now want to bound $R_{0}\left(b^{-2^{n}}\right)$ precisely. We have

$$
R_{0}(x)=Q_{0}(x) \mathbf{T}_{2}(x)-P_{0}(x)=\left(1-x^{2}\right) \mathbf{T}_{2}(x)-\left(1+x-x^{2}\right)=\mathcal{O}\left(x^{5}\right)
$$




\section{Boris AdAmczeWsin and TANGuy Rivoal}

and we see that the term of the power series $\left(1-x^{2}\right) \mathbf{T}_{2}(x)$ is $t_{n+2}-t_{n}$, which belongs to $\{-1,0,1\}$ because $t_{n} \in\{0,1\}$. Hence, it follows that

$$
\left|R_{0}(x)\right| \leqslant|x|^{5} \sum_{k=0}^{\infty}|x|^{k}=\frac{|x|^{5}}{1-|x|}
$$

and finally that $\left|R_{0}\left(b^{-2^{n}}\right)\right| \leqslant \frac{1}{b^{5 \cdot 2^{n}}} \cdot \frac{1}{1-b^{-2^{n}}}$.

Consequently, after some simplifications, we obtain that, for $n \geqslant 1$,

$$
\left|D_{n}\right| \leqslant \frac{1}{b}\left(1-\frac{1}{b}\right)\left(1+\frac{1}{b^{2^{n}}}\right) \prod_{j=1}^{n-1}\left(1-\frac{1}{b^{2^{j}}}\right)
$$

(For $n=1$, the value of the empty product is 1 .) We now observe that $(1-1 / b)\left(1+1 / b^{2^{n}}\right)<1$ for any $n \geqslant 1$ and that the product on the right hand side of (14) is always $\leqslant 1$. Therefore, we have proved that $\left|D_{n}\right|<1 / b \leqslant 1 / 2$ for any $n \geqslant 1$, as claimed. This finishes the proof of the theorem.

Numerically, it seems that for any integer $b \geqslant 2$ and any $n \geqslant 1, p_{n}(b)$ and $q_{n}(b)$ are coprime but at this point we do not know how to prove this. If this is true, the assumptions $(i),(i i)$ and $(i i i)$ in lemma 4.1 would then be fulfilled by the sequences $p_{n}(b)$ and $q_{n}(b)$ and this would imply the following

Conjecture 5.2. For any integer $b \geqslant 2$, we have $\mu\left(\mathbf{T}_{2}(1 / b)\right) \leqslant 3$.

\section{REFERENCES}

1 B. Adamczewski \& Y. Bugeaud, On the complexity of algebraic numbers I. Expansions in integer bases. Ann. of Math. 165 (2007), 547-565.

2 B. Adamczewski \& Y. Bugeaud, Mesures de transcendance et aspects quantitatifs de la méthode de Thue-Siegel-Roth-Schmidt, Preprint 2007.

3 B. Adamczewski, Y. Bugeaud \& F. Luca, Sur la complexité des nombres algébriques, C. R. Acad. Sci. paris 339 (2004), 11-14.

4 B. Adamczewski \& J. Cassaigne, Diophantine properties of real numbers generated by finite automata, Compos. Math. 142 (2006), 1351-1372.

5 J.-P. Allouche \& J. O. Shallit, Automatic Sequences: Theory, Applications, Generalizations, Cambridge University Press, 2003.

6 Y. Bugeaud, Diophantine approximation and Cantor sets. Math. Ann. 341 (2008), 677-684.

7 A. Cobham, Uniform tag sequences, Math. Systems Theory 6 (1972), 164-192.

8 G. Christol, Ensembles presques périodiques k-reconnaissables, Theoret. Comput. Sci. 9 (1979), 141-145.

9 G. A. Baker and P. Graves-Morris, Padé approximants, Second edition, Encyclopedia of Mathematics and its Applications 59, Cambridge University Press, 1996.

10 J. H. Loxton \& A. J. van der Poorten, Arithmetic properties of automata: regular sequences, J. reine angew. Math. 392 (1988), 57-69.

11 A. J. Kempner, On transcendental numbers, Trans. Amer. Math. Soc. 17 (1916), 476-482.

12 S. Lehr, Sums and rational multiples of $q$-automatic sequences are $q$-automatic, Theoret. Comput. Sci. 108 (1993), 385-391.

13 M. Lothaire, Algebraic Combinatorics on Words, Vol. 90 of Encyclopedia of Mathematics and Its Applications. Cambridge University Press, 2002.

14 K. Mahler, Arithmetische Eigenschaften der Lösungen einer Klasse von Funktionalgleichungen, Math. Ann. 101 (1929), 342-366. Corrigendum 103 (1930), 532.

15 T. Rivoal, Convergents and irrationality measures of logarithms, Rev. Mat. Iberoamericana 23.3 (2007), 931-952. 
IRRATIONALITY MEASURES FOR SOME AUTOMATIC REAL NUMBERS

16 J. O. Shallit, Number theory and formal languages, in Emerging applications of number theory, IMA Volumes (Springer, Berlin, 1999), 547-570.

Boris Adamczewski Boris.Adamczewski@math.univ-lyon1.fr

CNRS, Université de Lyon, Université Lyon 1, Institut Camille Jordan, 43 boulevard du 11 novembre 1918, 69622 Villeurbanne Cedex, France

Tanguy Rivoal rivoal@ujf-grenoble.fr

CNRS, Université Grenoble I, Institut Fourier, 100 rue des Maths, BP 74, 38402 Saint-Martind'Hères cedex, France 